\title{
Editorial
}

\section{Nanomaterials for Energy-Efficient Applications}

\author{
Xiaohu Huang, ${ }^{1}$ Guozhong Xing, ${ }^{2}$ Yongfeng $\mathrm{Li}^{3}{ }^{3}$ and Ekaterina Nannen ${ }^{4}$ \\ ${ }^{1}$ Institute of Materials Research and Engineering, Agency for Science, Technology and Research (A*STAR), 3 Research Link, \\ Singapore 117602 \\ ${ }^{2}$ School of Materials Science and Engineering, The University of New South Wales, Sydney, NSW 2052, Australia \\ ${ }^{3}$ Key Laboratory of Physics and Technology for Advanced Batteries (Ministry of Education), College of Physics, Jilin University, \\ Changchun 130012, China \\ ${ }^{4}$ Department of Electrical Engineering and Information Technology, University of Duisburg-Essen, 47057 Duisburg, Germany
}

Correspondence should be addressed to Xiaohu Huang; xhhuang.nano@gmail.com

Received 4 December 2014; Accepted 4 December 2014

Copyright (C) 2015 Xiaohu Huang et al. This is an open access article distributed under the Creative Commons Attribution License, which permits unrestricted use, distribution, and reproduction in any medium, provided the original work is properly cited.

The ways we used to generate and utilize energy have been threatening our living environment. For example, the crude oil spills in Gulf of Mexico in 2010 left devastating effects to the Gulf ecosystem, Fukushima nuclear plant leaks in Japan in 2012 threatened the lives of thousands of people, and the Haze in the cities of China at present affects the health of millions of people. All of them remind us of the global energy and environment crisis that we have to face in the near future. Alternative solutions in energy generation and utilization have to be explored towards a sustainable earth.

Many breakthroughs in the area of nanomaterials and nanosciences have been reported recently [1-12], which open up tremendous opportunities to tackle the challenges in energy and environment. With shrinking the size of the materials into nanoscale, fewer materials are required, which translates to higher utility efficiency of the resources. Moreover, with the dimensions of the materials decreasing to a scale comparable to the characteristic length of the physical properties, behaviors of photon emission [1], electron transport [2], surface train [3], and phonon scattering [4] change drastically compared to their bulk counterpart. The resulting properties have very important implications to their applications in high brightness light-emitting diodes [5-7], low power nanoelectronics [2], high efficiency solar cells [8], thermoelectric power generator [4], and so forth. In addition, the large surface-to-volume ratio in nanomaterials facilitates more efficient chemical reactions, which is highly desirable in many applications such as high performance batteries and catalysts $[9,10]$. Therefore, the research activities of nanomaterials on energy-related applications have surged over the past decade, which is manifested by the exponential growth of the corresponding research publications and citations as shown in Figure 1. It is very clear that the research on energyrelated nanoscience and nanotechnology has been becoming extremely active since 2010 .

However, the success of nanomaterials in energy-efficient applications relies on our ability to synthesize nanomaterials at will and to understand and tailor the properties of the nanomaterials $[11,12]$ and to integrate the nanomaterials into devices $[1,2,7,9]$. This special issue solicits some of these topics to foster the applications of nanomaterials in the emerging energy research. Moreover, the research trend of nanomaterials towards high performance energy-efficient applications could be summarized from the articles published in this special issue, for example, chemical doping, interface engineering, and hybridizing.

We hope that the readers will find in this special issue not only the interesting results but also important questions to be resolved and finally incorporate the findings into their own research. We hope this special issue can inspire more 


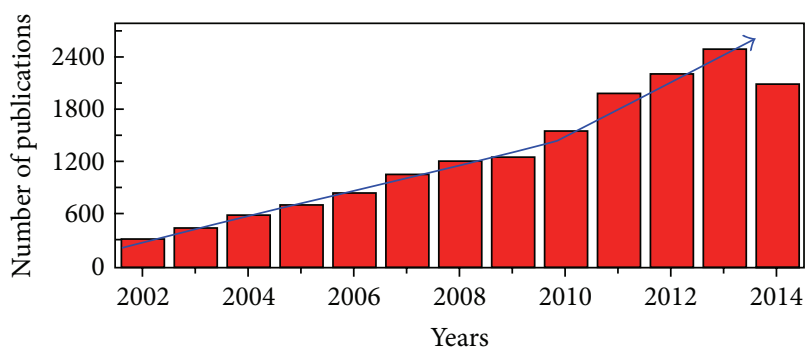

(a)

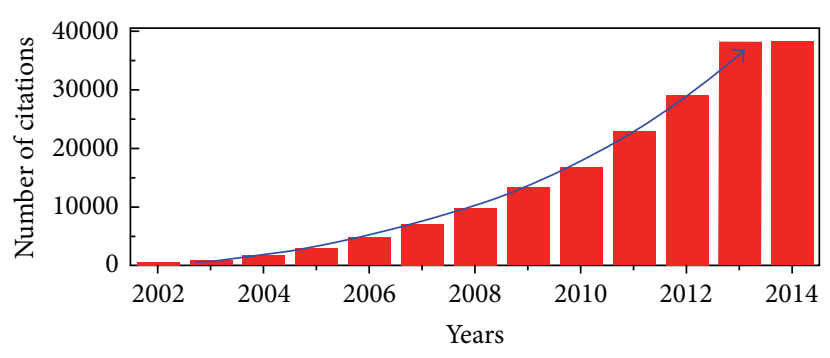

(b)

Figure 1: The numbers of (a) publications and (b) citations retrieved from Web of Science by listing both "nano" and "energy" as the key words in the search engine. The search was conducted on November 21, 2014; thus any update after that was not reflected in this figure.

research efforts on nanomaterials towards energy-efficient and environmentally friendly applications.

\section{Xiaohu Huang \\ Guozhong Xing \\ Yongfeng Li \\ Ekaterina Nannen}

[11] X. Huang, L. Li, X. Luo, X. Zhu, and G. Li, "Orientationcontrolled synthesis and ferromagnetism of single crystalline Co nanowire arrays," The Journal of Physical Chemistry C, vol. 112, no. 5, pp. 1468-1472, 2008.

[12] G. Z. Xing, X. S. Fang, X. Huang et al., "Ultrathin single-crystal $\mathrm{ZnO}$ nanobelts: Ag-catalyzed growth, optical absorption and field emission properties," Nanotechnology, vol. 21, Article ID 255701, 2010.

\section{References}

[1] Y. Li, W. Yin, R. Deng et al., "Realizing a $\mathrm{SnO}_{2}$-based ultraviolet light-emitting diode via breaking the dipole-forbidden rule," NPG Asia Materials, vol. 4, no. 11, article e30, 2012.

[2] W. Lu and C. M. Lieber, "Nanoelectronics from the bottom up," Nature Materials, vol. 6, no. 11, pp. 841-850, 2007.

[3] X. Huang, G. Li, L. B. Kong, Y. Z. Huang, and T. Wu, "Anisotropic surface strain in single crystalline cobalt nanowires and its impact on the diameter-dependent Young's modulus," Nanoscale, vol. 5, no. 23, pp. 11643-11648, 2013.

[4] D. Li, Y. Wu, P. Kim, L. Shi, P. Yang, and A. Majumdar, “Thermal conductivity of individual silicon nanowires," Applied Physics Letters, vol. 83, no. 14, pp. 2934-2936, 2003.

[5] X. H. Huang, Z. Y. Zhan, K. P. Pramoda, C. Zhang, L. X. Zheng, and S. J. Chua, "Correlating the enhancement of UV luminescence from solution-grown $\mathrm{ZnO}$ nanorods with hydrogen doping," CrystEngComm, vol. 14, no. 16, pp. 51635165, 2012.

[6] X. H. Huang, C. Zhang, C. B. Tay, T. Venkatesan, and S. J. Chua, "Green luminescence from $\mathrm{Cu}$-doped $\mathrm{ZnO}$ nanorods: role of Zn vacancies and negative thermal quenching," Applied Physics Letters, vol. 102, no. 11, Article ID 111106, 2013.

[7] E. Neshataeva, T. Kümmell, G. Bacher, and A. Ebbers, "Allinorganic light emitting device based on $\mathrm{ZnO}$ nanoparticles," Applied Physics Letters, vol. 94, no. 9, Article ID 091115, 2009.

[8] C. F. Guo, T. Y. Sun, F. Cao, Q. Liu, and Z. F. Ren, "Metallic nanostructures for light trapping in energy-harvesting devices," Light: Science and Applications, vol. 3, article e161, 2014.

[9] G. Z. Xing, Y. Wang, J. I. Wong, Y. M. Shi, S. Li, and H. Y. Yang, "Hybrid $\mathrm{CuO} / \mathrm{SnO}_{2}$ nanocomposites: towards costeffective and high performance binder free lithium ion batteries anode materials," Applied Physics Letters, vol. 105, Article ID 143905, 2014.

[10] H. R. Byon, J. Suntivich, and Y. Shao-Horn, "Graphene-based non-noble-metal catalysts for oxygen reduction reaction in acid," Chemistry of Materials, vol. 23, no. 15, pp. 3421-3428, 2011. 

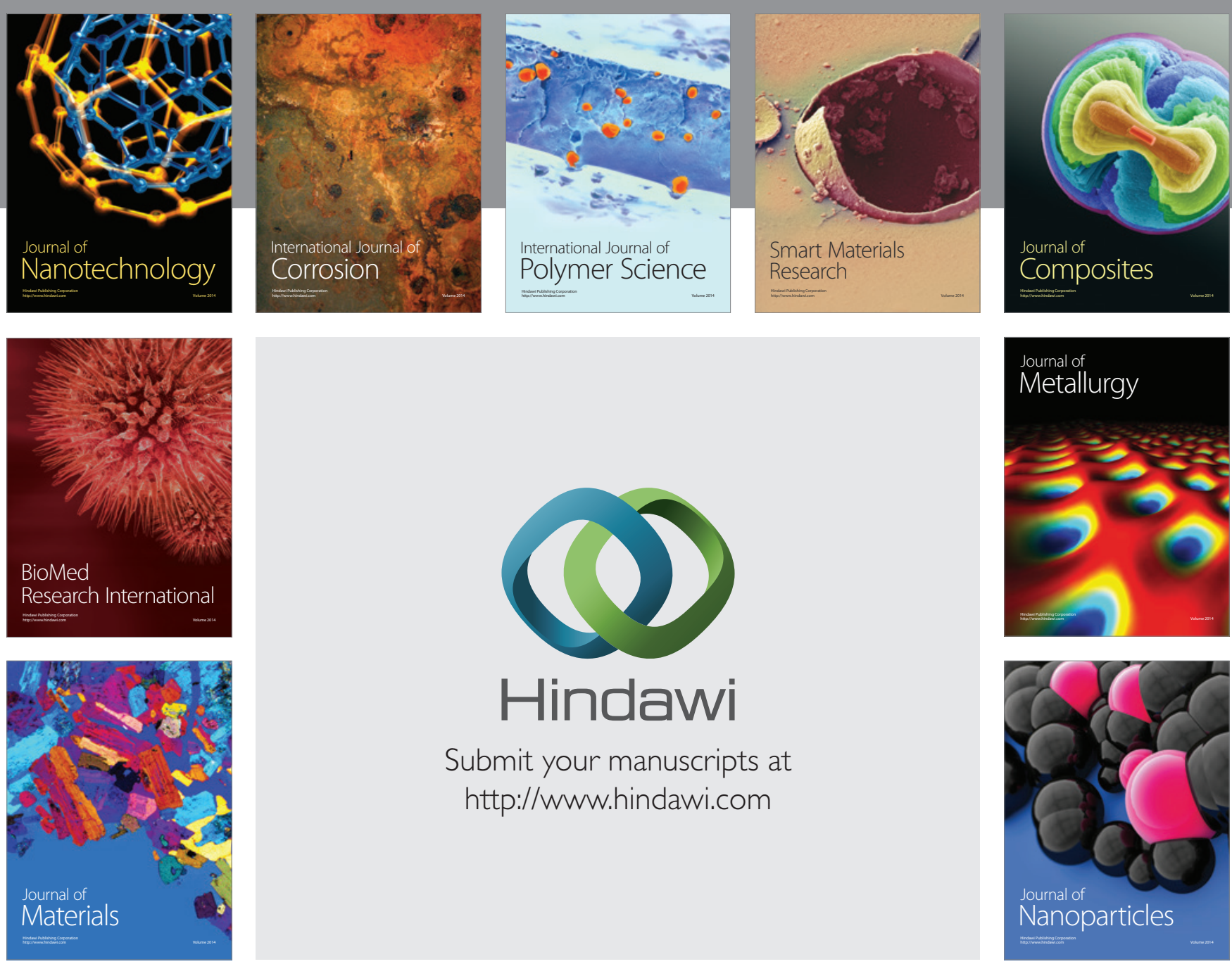

Submit your manuscripts at http://www.hindawi.com
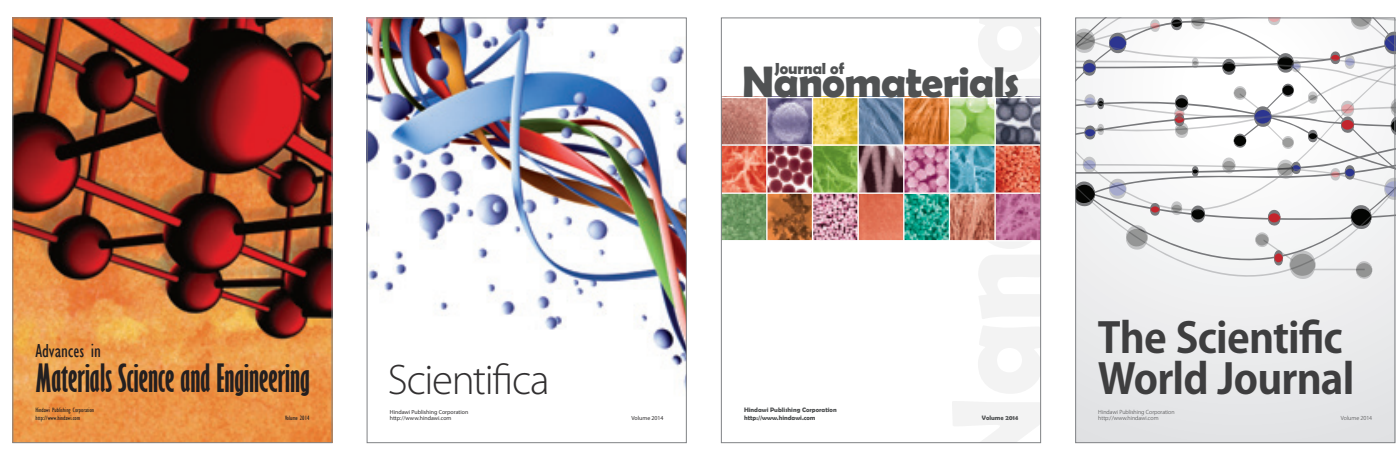

\section{The Scientific World Journal}
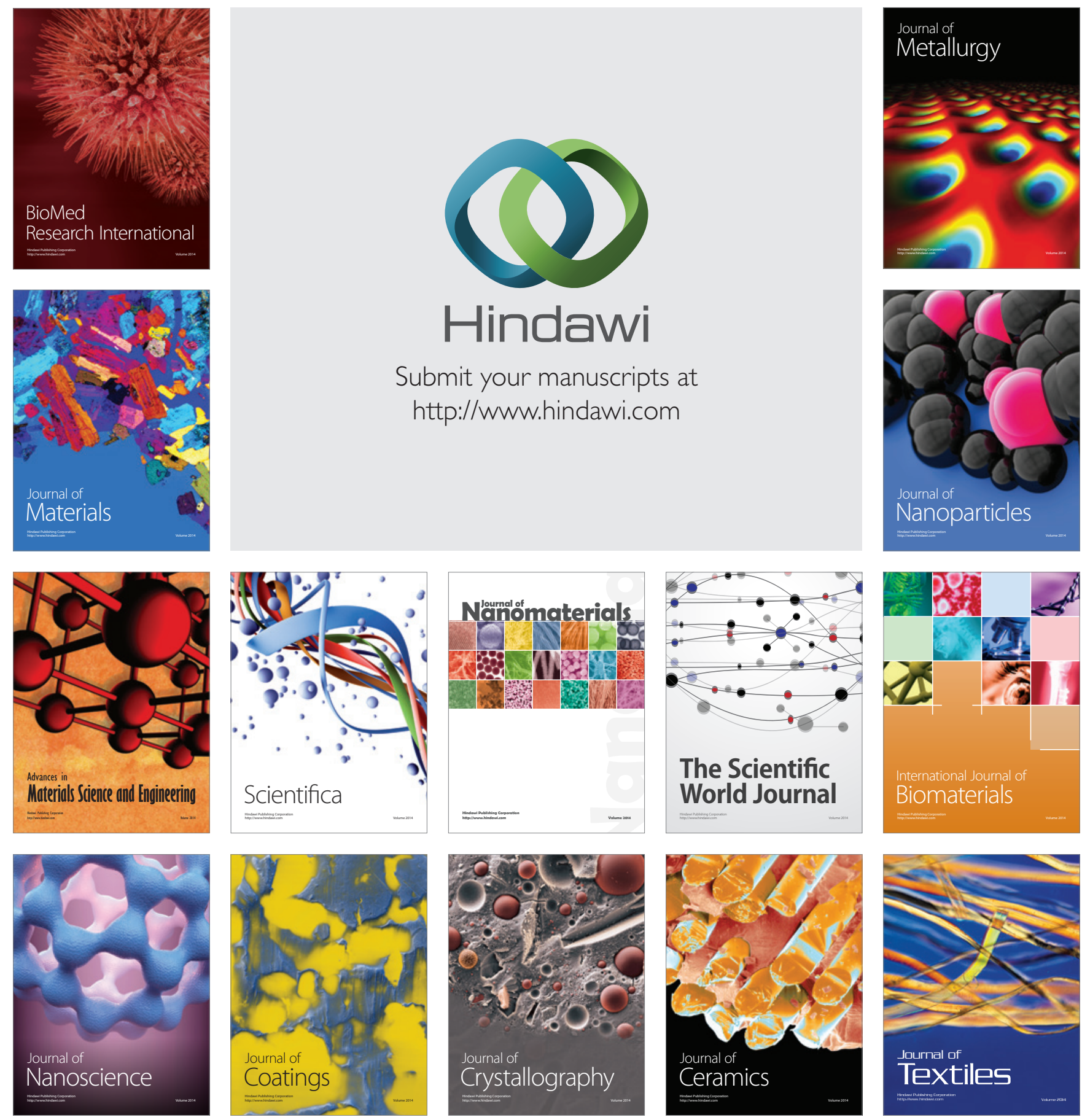